\title{
Kernos
}

Revue internationale et pluridisciplinaire de religion grecque antique

$2 \mid 1989$

Varia

\section{Rituel funéraire et coût des obsèques en Grèce classique}

\section{Xavier De Schutter}

\section{OpenEdition}

\section{Journals}

Édition électronique

URL : http://journals.openedition.org/kernos/234

DOI : $10.4000 /$ kernos.234

ISSN : 2034-7871

\section{Éditeur}

Centre international d'étude de la religion grecque antique

\section{Édition imprimée}

Date de publication : 1 janvier 1989

Pagination : 53-66

ISSN : 0776-3824

\section{Référence électronique}

Xavier De Schutter, «Rituel funéraire et coût des obsèques en Grèce classique », Kernos [En ligne],

2 | 1989, mis en ligne le 02 mars 2011, consulté le 02 mai 2019. URL : http://journals.openedition.org/ kernos/234 ; DOI : 10.4000/kernos.234 
Kernos, 2 (1989), p. 53-66.

\section{RITUEL FUNÉRAIRE ET COÛT DES OBSÈQUES EN GRÈCE À L'ÉPOQUE CLASSIQUE*}

Se faire enterrer coûte cher. Il est d'ailleurs possible de nos jours de souscrire à une assurance dans le but d'épargner à ses proches les dépenses inévitables des obsèques, telles que la location d'un corbillard et l'achat d'un cercueil $^{1}$. D'autre part, les ethnologues ont maintes fois constaté que les funérailles donnent lieu, dans les civilisations de culture archaïque, à des cérémonies collectives où les dépenses ostentatoires constituent un élément indispensable. Les festivités peuvent étaler sur plusieurs jours un grand déploiement de sacrifices et d'offrandes, de chants, de danses, de parures, de cortèges, de banquets, etc. La famille endeuillée qui assume les frais est souvent endettée ou réduite à la misère après de telles solennités. Ainsi, le veut le groupe social qui «affirme son identité et sa pérennité par le médiat de ses morts ${ }^{2}$. On le sait depuis l'analyse d'Émile Durkheim, les funérailles sont une période de forte socialisation ${ }^{3}$ : la disparition d'un membre de la famille resserre les liens familiaux entre les survivants, et celle d'un leader du peuple entraîne des funérailles nationales qui ont le même effet.

Dans ces notes, il ne sera question que des funérailles privées de la Grèce classique : je n'aborderai pas ici les cérémonies grandioses célébrées en

* M. Guy Donnay et Mme Annie Verbanck ont bien voulu relire ces notes et les enrichir de leurs suggestions. Qu'ils soient assurés de ma sincère reconnaissance.

1 Il existe aussi dans nos sociétés occidentales des pratiques funéraires réservées aux seuls fortunés, telles que la thanatopraxie et surtout la cryogénisation, cf. L.V. Thomas, Le cadavre. De la biologie à l'anthropologie, 1980, p. 128 sq.; 152 sq.; 155 : «en 1977 , les tarifs (de la cryogénisation) atteignaient 7750 dollars pour le traitement du corps et 2500 dollars par an pour les frais de conservation de la capsule dans laquelle il faut constamment renouveler l'azote liquide".

2 L.V. Thomas, op. cit., p. 195-196, et Rites de mort, 1985, p. 228-235 : «le parti pris d'ostentation que supposent les gros frais d'obsèques se justifie par l'intention de valoriser le défunt, de lui rendre $1^{\prime}$ hommage qui lui est dû, mais aussi de proclamer l'intime cohésion de la famille et des amis plus unis donc plus vivants que jamais autour du disparu».

3 E. DuRKHEIM, Les formes élémentaires de la vie religieuse, 1912, p. 557 sq. et surtout p. 571-574; 589 : les manifestations de deuil compensent l'affaiblissement provoqué par la mort d'un membre de la collectivité, et permettent même au groupe de sortir renforcé de l'épreuve. 
l'honneur des héros de l'épopée homérique ${ }^{4}$ ni des soldats morts pour la patrie et enterrés en grande pompe aux frais de l'État ${ }^{5}$; je ne parlerai pas non plus de la richesse bien connue des tombes royales de l'époque mycénienne ni du rituel funéraire particulier auquel avaient droit les rois de Sparte ${ }^{6}$.

Les dépenses sont d'autant plus élevées que les derniers devoirs ne se limitent pas aux seules funérailles, entendons par là les rites observés depuis le décès jusqu'à la mise au tombeau, mais se prolongent encore par des sacrifices ou offrandes posthumes à l'occasion de fêtes commémoratives. La Grèce a connu ces rites célébrés aux dates anniversaires de la mort ( $\tau$ d̀

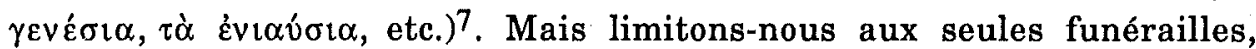
premier acquittement d'une série de soins pieux auxquels ont droit les défunts.

L'iconographie des vases funéraires et les textes montrent que les

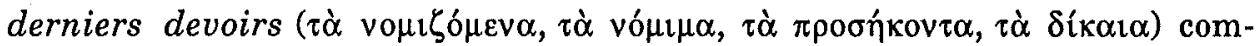
mencent par la toilette funèbre dont se chargent les femmes de la maisonnée. Le corps est lavé, parfumé, couronné et habillé. Aujourd'hui encore, surtout dans nos campagnes, le mort est exposé dans son «costume du dimanche». Que cette toilette qui précède l'exposition du mort ait pu être luxueuse, les peintures sur vases le montrent clairement, depuis les cratères de l'art géométrique jusqu'aux lécythes à fond blanc de l'époque classique. Or, précisément, une inscription nous apprend que le coût des habits du mort a pu

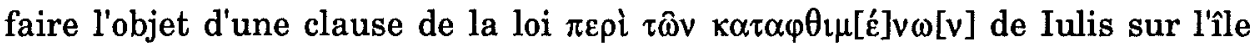
de Céos. Le texte date de la fin du Ve siècle et s'inspire de la législation solonienne ${ }^{8}$. Il prescrit «d'enterrer le mort ainsi : dans trois vêtements

4 La mort, les morts dans les sociétés anciennes, sous la dir. de J.P. VERNANT et G. GNOLI, 1982, p. 27-88 : articles de N. LoraUX, J.P. VERnANT ET A. SchNaPPGOURBEILLON.

5 THUC., II, 34; cf. aussi N. LoRAUX, L'invention d'Athènes. Histoire de l'oraison funèbre dans la cité classique, 1981. Il arrivait même que l'on promette aux soldats des funérailles grandioses pour les inciter à la vaillance; cf. XÉN., Hell., VI, 1, 6.

6 Après sa mort, le roi spartiate était honoré comme un demi-dieu; cf. XÉN., Rép. des Lacéd., 15, 9; mort à l'étranger, il était embaumé et rapatrié; cf. XÉn., Hell., V, 3, 19; Agés., XI; HDT, VI, 58-59; PLUT., Agés., 40, 4. Mis à part ce cas très spécifique des rois de Sparte, la Grèce n'a guère pratiqué avant l'époque hellénistique la divinisation des souverains (ce qui suppose des funérailles extravagantes), comme ce fut le cas en Égypte pharaonique et dans la Rome impériale. A titre d'exemple, notons que les obsèques de l'empereur Vespasien coûtèrent dix millions de sesterces; cf. Sútr., Vesp., 19.

7 Dans le discours C. Spoudias de Démosthène, il est question d'une mine d'argent dépensée lors des Némésies en l'honneur d'un père décédé.

8 DiTT., Syll. ${ }^{3}, 1218,1.1-6$; F. SoKolowsKi, Lois sacrées des cités grecques, $1969, \mathrm{n}^{\circ} 97$. 
blancs ${ }^{9}$, ou moins, le strôma, l'endyma et l'épiblèma, d'une valeur totale qui

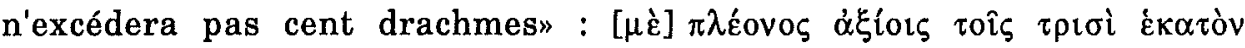
$\delta \rho[\alpha \chi] \mu \varepsilon ́ \omega v$.

Plusieurs lois dans l'Antiquité ont visé à restreindre le luxe des funérailles car les dépenses somptueuses auxquelles donnaient lieu les enterrements étaient l'occasion pour l'aristocratie d'exalter le prestige des siens ${ }^{10}$. Entre autres manifestations ostentatoires, la loi de Solon s'efforçait de limiter les lamentations funèbres qui avaient lieu auprès du lit durant la prothésis, mais elle interdisait surtout les thrènes composés à l'avance ( $\tau$ ò

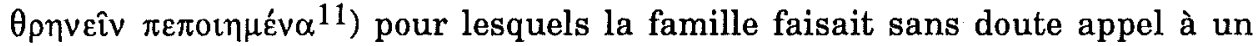
poète ou à des pleureuses professionnelles. Lorsque ces chanteurs ou musiciens funèbres étaient des esclaves, des Cariens par exemple ${ }^{12}$, leur location devait être d'un prix modique, mais il est évident que seules les familles riches pouvaient commander des thrènes aux grands poètes qui s'illustrèrent dans le genre, tel que Pindare.

Après la prothésis, qui durait vraisemblablement un jour ${ }^{13}$, le corps était emmené en grande pompe vers sa dernière demeure. Du moyen de transport utilisé depuis la maison mortuaire jusqu'au cimetière, simple brancard porté à dos d'homme ${ }^{14}$ ou char luxueux tiré par deux ou quatre chevaux ${ }^{15}$, dépendait le coût de l'ekphora.

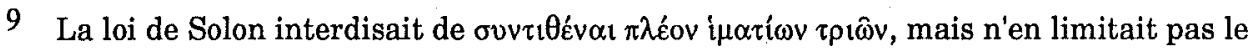
prix. Du moins Plutarque, dont nous sommes tributaires pour la connaissance de cette réforme, n'en parle pas, cf. PLUT., Sol., 21, 6. Il est difficile de déterminer avec précision ce que le texte entend par $\sigma \tau \rho \hat{\omega} \mu \alpha$, $\varepsilon^{\prime} v \delta \mu \alpha$ et $\dot{\varepsilon} \pi \dot{\beta} \beta \lambda \eta \mu \alpha$ : le premier est peutêtre un tapis sur lequel est étendu le défunt; le second est son habit proprement dit; le troisième doit être la couverture qui recouvre le mort ou la bière tout entière, c'est-àdire le linceul. La loi des Labyades de Delphes (ca. 400) précise qu'il n'y aura qu'un seul strôma; cf. F. SoKoLowsKI, op. cit., $\mathrm{n}^{\circ}$ 77, 1. 11-12.

10 L'ensemble de la réforme de Solon visait à rompre la solidarité du genos; cf. L. GERNET-A. BOULANGER, Le génie grec dans la religion, 1932, p. 137-138; J.-P. VERnANT, Les origines de la pensée grecque, 1962, p. 60-69. Comme le dit Cicéron, le but de ces mesures était de tolli fortunae discrimen in morte, cf. CIC., De leg., II, 23, 59.

11 PluT., Sol., 21, 6 : ce sont des «lamentations versifiées» de caractère aristocratique, cf. N. LoRAUX, op. cit., p. 44 sq. et p. 371, n. 186. Homère déjà parle d'un chanteur

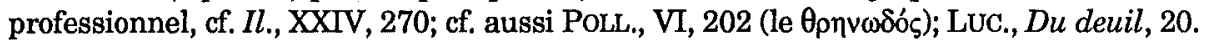

12 Platon fait allusion à ces «gens qu'on loue pour escorter les morts aux accents de la

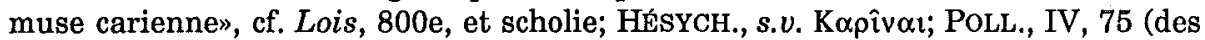
flûtistes).

13 DÉm., C. Macart., 62; ANTIPH., Sur le chor., 34.

14 Le brancard était sans doute porté par des parents, ou éventuellement par des

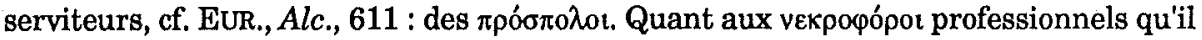


A l'époque classique, les deux pratiques de l'inhumation et de l'incinération se concurrençaient, mais il est vraisemblable qu'en raison de la rareté du bois en Attique la crémation ait été plus onéreuse et donc plus estimée par les riches Athéniens ${ }^{16}$.

Le mort emportait dans son tombeau un certain nombre de ses ktèmata, objets favoris et utilitaires qui lui appartenaient en propre : strigile, miroir, alabastre, bijoux, jouets, arme, lampe, etc. ${ }^{17}$. A partir de la fin du VIe siècle, on plaçait entre ses dents l'obole destiné à payer le nocher Charon et, à ses côtés, le gâteau de miel qui devait apaiser les crocs de Cerbère ${ }^{18}$. Il recevait aussi de quoi se nourrir et se désaltérer dans l'au-delà ${ }^{19}$. Ici encore la loi

fallait payer, ils n'apparaissent dans nos sources qu'à l'époque romaine; cf. PoL., VII, 195; PLUT., Cat. maj., 9, 2; Anth. pal., VII, 634; R. GARLAND, The Greek way of death, 1985, p. $34 ; 143$.

15 Le char était un élément de la pompe aristocratique; cf. D.C. KURTZ-J. BOARDMAN, Greek burial customs, 1971, p. 60; pl. 4-5; 16; p. 145; 149; pl. 34-35; M. ALEXIOU, The ritual lament in Greek tradition, 1974, p. 7, n. 31.

16 S.C. HUMPHREYS, Family tombs and tomb cult in ancient Athens, in JHS, 100 (1980), p. 100; R. GARLAND, A first catalogue of Attic peribolos tombs, in ABSA, 77 (1982), p. 131 et n. 30. Mais il y a lieu de nuancer car, comme le remarque R. GARLAND, The Greek way of death, p. 34, certaines tombes à inhumation de l'époque archaïque renfermaient les offrandes les plus luxueuses.

17 D.C. KURTZ-J. BOARDMAN, op. cit., p. 76-79 (ép. archaïque); p. 100-102 (ép. classique); p. 203-217.

18 L'obole de Charon apparaît pour la première fois dans les textes chez Aristophane, cf. Gren., 140 (et scholie); 270. Sur l'origine de Charon et de cet usage, cf. E. ROHDE, Psyché, $1928^{10}$ (trad. franç.), p. 20-21; 252 et n. 3 : Rohde a sans doute raison de voir dans cette pièce de monnaie «un reste symbolique de l'offrande d'autrefois, où l'on devait donner au mort l'ensemble de ses biens». Ce serait une pars pro toto. Dans ce cas, le prix qu'il fallait payer pour passer le fleuve infernal serait un mythe étiologique visant à expliquer cette pratique devenue incompréhensible. L'opinion de Rohde est reprise par H. BORZA, Le mythe de l'obole à Charon, in Orbis, 4, 1 (1955), p. 134-148. Cf. aussi D.C. KURTZ-J. BoARDMAN, op. cit., p. 166; 211; pl. 40; E. VERMEULE, Aspects of death in early Greek art and poetry, 1979; p. 4; 211, n. 7; 70-72; 229, n. 57; W. BURKERT, Greek religion, 1985, p. 197; 427, n. 21. Sur le gâteau de Cerbère, cf. ARISTOPH., Nuées, 507; Lys., 601-602 (et scholie); E. ROHDE, op. cit., p. 251, et pour tout ce qui concerne le Totenteil, cf. E.F. BRUCK, Totenteil und Seelgerät im griechischen Recht, in $M B P, 9$ (1926), en part. p. 139; 145-146.

19 Sur les vases et la nourriture dans les tombes, cf. D.C. KURTZ-J. BOARDMAN, op. cit., p. $145 ; 149-161 ; 204-207 ; 215$ (os d'animaux, etc.). Sur la soif des morts, cf. W. DEONNA, Croyances funéraires, in RHR, 119 (1939), p. 53-77; cf. encore E.R. DoDDs, Les Grecs et l'irrationnel, $1977^{2}$ (trad. franç.), p. 140-141. 
intervint pour limiter cette part du mort : à Céos, il était interdit «d'apporter au tombeau plus de trois conges de vin et plus d'un conge d'huile ${ }^{20}$ ». A Sparte, la loi était plus sévère : Lycurgue avait défendu de «rien enterrer avec

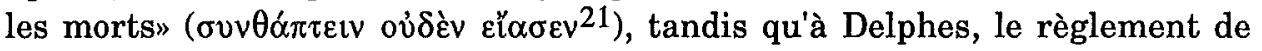
la phratrie des Labyades fixait la valeur maximale des offrandes à trente-

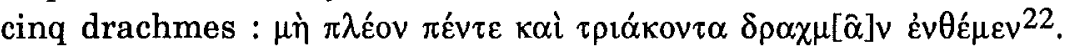

Le vin et l'huile dont il est question dans la loi de Céos devaient servir à la libation faite sur le tombeau ${ }^{23}$ en même temps qu'un sacrifice :

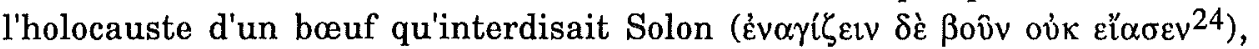
mais que la loi de Céos autorisa "selon la coutume des ancêtres" ( $\pi \rho \circ \sigma \varphi \alpha \gamma i$ í

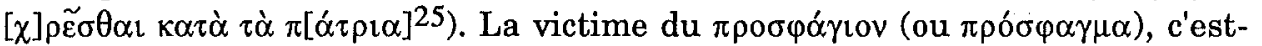
à-dire du sacrifice avant l'enterrement ${ }^{26}$, était un bœu ${ }^{27}$ ou un mouton ${ }^{28}$; dans l'un et l'autre cas, ce sacrifice n'était sans doute accompli que par les gens aisés. Je reviendrai sur le prix de ces victimes.

Enfin, un repas, le $\pi \varepsilon \rho i \delta \varepsilon i \pi v o v$, clôturait les funérailles. La cohésion familiale, que la cérémonie funèbre revitalise au moment où elle est le plus menacée, trouvait dans ce repas l'occasion de s'affirmer pleinement : la réunion des membres de la famille au retour du cimetière est le moment fort des funérailles. Ici encore, les ethnologues nous décrivent de véritables festins copieusement arrosés qui ont le caractère du potlach. Le repas est en effet l'acte de communion par excellence. Il "réalise entre convives une

20 DITT., $S y l l^{3}$. 1218, 1. 8-9. Par mesure d'économie, il fallait aussi ramener à la maison les vases, le lit et les strômata; cf. 1. 10; 13-14.

21 PLUT., Lyc., 27, 2.

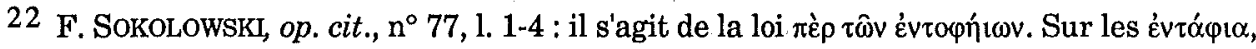

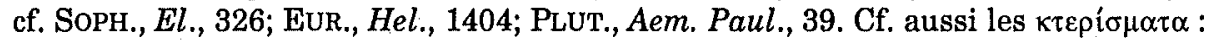
SoPH., $E d$. Col., 1410; El., 434; 931; Eur., Suppl., 309.

23 Les tragiques donnent de nombreux exemples de cette libation faite lors des funérailles ou ultérieurement, cf. EscH., Pers., 607 sq. (libation de lait, miel, eau, vin et offrandes d'olives et de fleurs); Chœh., 14-15; 87-99; 109 sq.; 484-488: EuR., Or., 112 sq.; 1320-1322; Iph. Taur., 156 sq.; 653 sq.; SoPH., Ant., 430-431; 891 sq. etc.

24 PluT., Sol., 21, 6.

25 DiTT., Syll ${ }^{3}$., 1218, 1. 13.

26 Signalons toutefois que, d'après PLAT., Min., 315c, aux temps anciens, le sacrifice avait

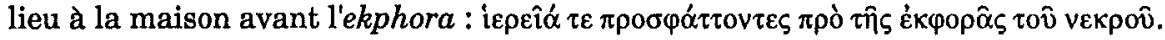

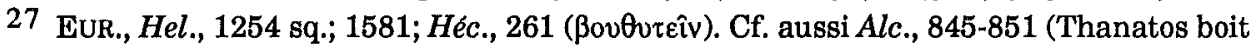
près de la tombe le sang des victimes); PLUT., Arist., 21 (un bœuf noir sacrifié pour les héros morts à Platées).

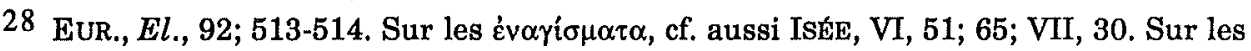
offrandes d'animaux aux époques mycénienne et homérique, cf. M. ANDRonIKos, Totenkult, in Arch. Hom., III, W, 1968, p. 87-91. 
identité d'être, une sorte de consanguinité", selon l'expression de J.-P. Vernant qui mentionne en outre que les membres de l'oîkoৎ sont

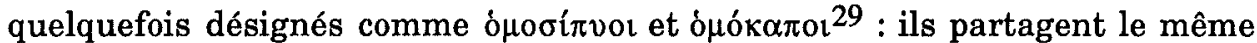
pain et mangent à la même table. A cela, j'ajouterais qu'ils partagent aussi

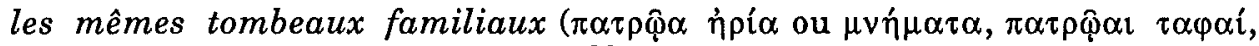
etc.) et sont donc en outre ó $\mu$ ó $\tau \alpha_{\varphi \circ \iota^{30}}{ }^{30}$. Ce rapprochement entre les commensaux et le culte funéraire est d'ailleurs renforcé par l'étymologie du terme $\tau \rho \alpha \dot{\alpha} \varepsilon \zeta \alpha$ qui désignait initialement toute surface plane sur laquelle on mangeait en diverses occasions et notamment lors du service funèbre ${ }^{31}$. Dans de nombreuses sociétés archaïques, le mort est censé prendre part au repas $^{32}$. En Grèce aussi, et en Attique depuis ca. 400 av. J.-C. ${ }^{33}$, des basreliefs représentent le mort accoudé sur une klinè et partageant un repas avec ses proches ${ }^{34}$, mais cette scène conventionnelle, baptisée par les archéologues sous le nom de Totenmahl, n'a très vraisemblablement rien à voir avec le perideipnon qui suit les funérailles ${ }^{35}$ et qui, dès Homère, se déroulait à la

29 J.-P. VERNANT, Les origines de la pensée grecque, 19835, p. 75; Mythe et pensée chez les Grecs, $1985^{3}$, p. 173.

30 Il apparaît dans une loi de Solon que les ó $\mu$ ó $\tau \alpha \varphi \circ$ forment une communauté comparable aux phratries ou aux thiases, cf. Digesta, XLVII, 22, 4 (= Corp. Iuris Civ., I, 195416, ed. P. Krueger et Th. Mommsen). Cf. aussi ESCH., I, 149, où Patrocle souhaite qu'Achille et lui-même soient ò $\mu$ ơớpous.

31 M. MuRKo, Das Grab als Tisch, in Wörter und Sachen, II, 1910, p. 79-160, en part. p. $120 \mathrm{sq}$. (chez les Slaves, le mot trapeza désigne toujours le banquet funéraire et les proches qui y participent).

32 L.V. Thomas, Le cadaure, p. 67-70 : «Le mort à tablew; Rites de mort, p. 160 : l'auteur considère le repas funéraire comme le substitut du repas cannibale.

33 R.N. THÖNGES-STRINGARIS, Das griechische Totenmahl, in MDAI(A), 80 (1965), p. 199, en part. $n^{\circ} 65$.

34 Les tentatives d'interprétation ont été nombreuses, mais pas toujours cohérentes : il y a souvent eu confusion entre reliefs votifs et stèles funéraires, ainsi qu'entre les offrandes faites au tombeau (holocauste) et le repas pris par les survivants après les funérailles. Pour l'état de la question et une bibliographie complète, cf. J.M. DENTZER, Le motif du banquet couché dans le Proche-Orient et le monde grec du VIIe au IVe siècle av J.-C., 1982 (BEFAR, 246), en part. p. 11-14; 529-557.

35 Nilsson souligne avec raison que rien n'indique que les Grecs de l'époque classique aient pensé que le défunt participait au repas; cf. M.P. NILSSON, Geschichte, I, 1955, p. 179. 
maison $^{36}$. Quoique l'on ne sache rien sur le «menu" du repas, il est clair qu'il devait mettre à contribution la famille endeuillée dans une mesure qui dépend évidemment de la teneur du repas et du nombre d'invités : une simple collation réservée à la famille proche ou un plantureux festin entre collatéraux et amis ${ }^{37}$. On connaît par exemple le cas des Bousélides, membres de plusieurs oî́or liés par la parenté, qui partageaient un $\mu v \hat{\eta} \mu \alpha$ Kotvóv ${ }^{38}$, et il ressort d'une allusion de Xénophon que les phratères prenaient part au deuil de leurs compagnons lors des Apaturies ${ }^{39}$. Il n'est donc pas exclu que le cercle des invités au perideipnon ait pu s'élargir au-delà des limites de la famille proche et que le repas ait quelquefois pris l'allure d'un véritable banquet. On comprend mieux dès lors pourquoi les lois imposaient le dispersement de la foule après l'ekphora ou après la mise au tombeau :

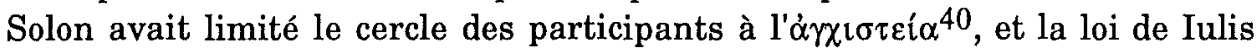

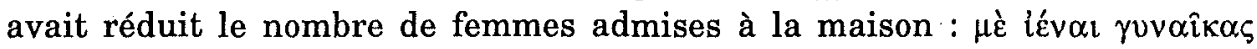

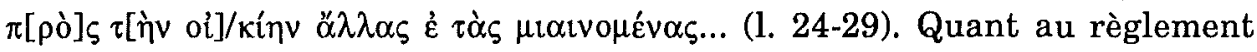
des Labyades, il ordonnait à chacun de s'en retourner chez soi, exception faite pour ceux qui vivent au même foyer et quelques autres proches parents :

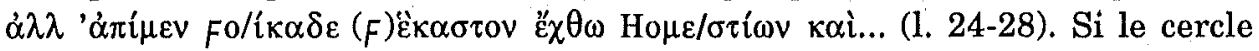

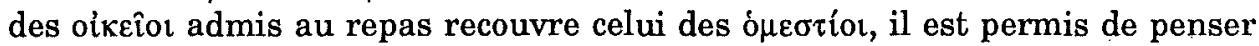
que le perideipnon désigne le repas «autour du foyer» plutôt que «autour du

36 Il., XXIV, 801-803 : le repas en l'honneur d'Hector dans le palais de Priam. Il est vrai que celui de Patrocle ( $I l$., XXIII, 28 sq.), a lieu près du bûcher et avant les funérailles, mais les funérailles de Patrocle, célébrées par une armée en campagne et dirigées par Achille en colère, présentent plusieurs anomalies, cf. M. ANDRONIKOS, op. cit., p. 15-18; A. Schnapp-Gourbeillon, Les funérailles de Patrocle, in La mort, les morts dans les sociétés anciennes, 1982 , p. 77-88.

37 THÉOPHR., Car., XIV, 7, fait allusion au stupide invité aux obsèques d'un ami. D'après ARTÉmID., Onirocr., V, 82, c'est le défunt qui offre l'hospitalité aux survivants.

38 DÉM., XLIII, 79.

39 Xén., Hell., I, 7, 8. Cela n'a rien d'étonnant vu que les phratères se réunissaient également pour fêter leurs confrères récemment mariés; cf. X. DE ScHUTTER, Le culte d'Apollon Patrôos à Athènes, in AC, 57 (1987), p. 106. Par ailleurs, Platon confirme que les phratries étaient concernées par les décès puisque, en tenant à jour les registres, elles prenaient note des naissances et des morts, cf. Lois, 785a.

40 DÉM., XLII, 62-63. 
tombeau» ${ }^{41}$. Le texte de Iulis nous apprend d'ailleurs qu'on procédait le lendemain des funérailles, après la purification de la maison, à un sacrifice

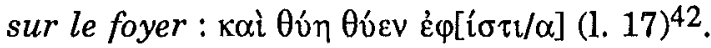

L'hestia, centre de l'habitat et gage de fixité pour la maisonnée, symbolise, avec sa flamme éternelle, la perpétuité de la famille. C'est sous le signe de la déesse Hestia que le groupe domestique affirme son unité lors des repas, et c'est autour de l'hestia que le nouveau-né est porté lors du rite des Amphidromies qui doit intégrer l'enfant dans l'oikos : le foyer n'est-il pas le lieu idéal pour un repas servi à l'occasion du décès d'un parent qui quitte cet oikos et gagne le monde des morts ${ }^{43}$ ?

Toilette et parures du mort, location de pleureuses, d'un chanteur de thrènes ou de fossoyeurs, achat d'un chariot funèbre et d'un cercueil, offrandes diverses déposées dans le tombeau, libations, sacrifices d'animaux et banquet funèbre : autant de dépenses auxquelles devait faire face la

41 W. BURKERT, Greek religion, 1985, p. 193, reprend l'interprétation traditionnelle qui voit dans le perideipnon un repas «autour du tombeau", ce qui l'oblige à imaginer une évolution - peu vraisemblable - dans le rite puisque, de toute évidence, à l'époque historique, le repas se déroulait à la maison mortuaire (ou peut-être parfois chez l'héritier, cf. ISÉE, VIII, 21-22). Déjà D.C. KURTZ et J. BOARDMAN, op. cit., p. 146-147, niaient la possibilité d'un repas pris au tombeau, mais sans en tirer la conclusion nécessaire quant à l'étymologie du mot; R. GARLAND, The Greek way of death, p. 3940, fait bien la distinction entre le perideipnon et les repas préparés à la tombe.

42 Par ailleurs, le terme ópokónor, qui désigne les commensaux de l'oikos, doit peut-être se

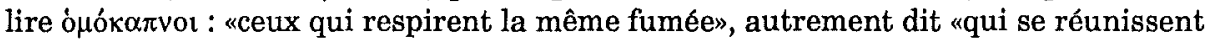
autour du même foyer", cf. ARIST., Pol., $1252 \mathrm{~b} 5$ (les mss $M$ et $P$ ); J.-P. VERnanT, Mythe et pensée, p. 173. N'oublions pas que le verbe $\dot{\sigma} \sigma \tau$ ó $\omega$ signifie «recevoir à sa

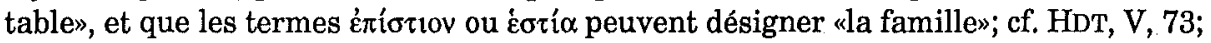
I, 176. Les ovveoríot sont ceux qui vivent sous le même toit; cf. EUR., Alc., 1151; PLAT., Euthyphr., 4b; ou qui participent au même festin; cf. EUR., $E l ., 784$; d'où les compagnons de table; cf. Anth. Pal., VI, 248.

43 Sur la symbolique de l'espace domestique, cf. J.-P. VERNANT, Mythe et pensée, p. 155-

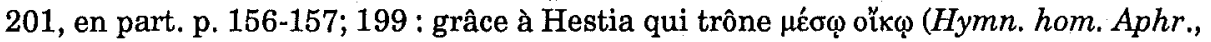
30), «la maison et le groupe familial entrent en contact avec le monde d'en bas». Notons que d'après PLAT., Min., 315d, les Grecs enterraient jadis leurs morts év oikia.. Pour Rome, cf. SERV., En., V, 64; VI, 152 (origine du culte des Lares et des Pénates). C'est en se basant sur ces indications que certains ont pensé jadis que le foyer domestique était à l'origine le symbole du culte des morts et que le tombeau du chef de famille devait se trouver à côté de l'hestia; cf. Fustel de CoulaNGES, La cité antique, 1864, 1. 1, chap. 3; E. RoHDE, op. cit, p. 188, n. 2. Dans cette optique, l'étymologie de perideipnon ne pose plus de problème vu que «autour du tombeau» revient à uautour du foyer». On se souviendra aussi de l'anecdote de PLUT., Phoc., 37, où les restes de Phocion sont confiés à l'hestia, et de la prière prononcée par Alceste

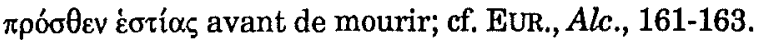


famille en deuil, autant de manifestations qui ont pu transformer les funérailles en une prétentieuse ostentation des richesses.

Mais il reste à parler de la dépense la plus importante de toutes, celle qui dut permettre le plus sûrement à l'aristocratie d'étaler au grand jour sa puissance : le monument funéraire. La tombe se prête d'autant mieux à cet emploi qu'elle est construite avec des matériaux durables et se dresse le long des routes ou dans les cimetières où son épitaphe et ses ornements apostrophent les passants. On ne s'étonnera pas que la loi athénienne ait tenté à plusieurs reprises de contrôler la tendance à la monumentalité de l'architecture funéraire ${ }^{44}:$ la loi de Solon fut suivie post aliquanto ${ }^{45}$ d'une seconde restriction (datée entre 510 et 480 ) et, en $317 / 6$, de celle de Démétrios de Phalère. Mais, en réalité, ce n'est qu'aux Ve et IIIe siècles que l'on constate une modération dans l'art funéraire; les monuments sculptés disparaissent à Athènes entre 480 et $430^{46}$, et après la réforme de Démétrios ${ }^{47}$. La loi post aliquanto interdisait de consacrer à la construction du sepulcrum plus que le travail de dix hommes pendant trois jours. Et Platon, dans ses Lois, défendait que le monticule de terre dépassât la hauteur que peut atteindre le travail de cinq hommes en cinq jours ${ }^{48}$ car, disait-il, «les funérailles les plus sobres sont les plus belles» 49 . Quant à Démétrios de Phalère, lorsqu'il dut à nouveau réglementer la construction des monuments, il limita leur hauteur à trois coudées. Ces restrictions en disent long sur le faste que devaient s'autoriser les riches, car même l'élévation d'un monument en marbre par dix hommes en trois jours pouvait rester, somme toute, assez chère. C'est surtout le type du peribolos burial que ces législateurs visaient : en effet, les tombeaux familiaux où reposaient plusieurs générations étaient les plus monumentaux et donc les plus coûteux ${ }^{50}$.

44 R. STUPPERICH, Staatsbegrabnis und Privatgrabmal im klassischen Athen, 1977, en part., p. 71 sq.; D.C. KURTZ-J. BOARDMAN, op. cit., p. 89-90; 121-122; 162; 166; 200-202; 363.

45 CIC, De leg., II, 26, 64.

46 Sur le rôle probable de l'iconographie des vases à cette époque, cf. S.C. HUMPHREYs, op. cit., p. 110 sq., 112 sq.

47 Les restrictions de Démétrios ont été appliquées, cf. D.C. KURTZ-J. BOARDMAN, op. cit., p. 162; 166-169; R. GARLAND, art. cit., in ABSA, 77 (1982), p. 127-128.

48 PLAT., Lois, XII, 958; CrC., De leg., 11, 27, 68.

49 PLAT., Lois, IV, 717d.

50 R. GARLAND, art. cit., p. 125-176, et en part. p. 130-132 : «Even a modest peribolos was an extremely costly undertaking and must have been well beyond the financial means of the majority of the population». En consequence de quoi, "some families unable to bear the financial burden of erecting a full-scale peribolos (...) were compelled to seek a cheaper alternative», par exemple un «peribolos substitute». 
En élevant de tels monuments, la famille exaltait moins la gloire du mort que sa propre puissance. En somme, même la mort ne résorbe pas les inégalités sociales, même la mort a une "histoire sociale» qui se lit dans les cimetières 51 . Dans son invective contre les injustices de la société athénienne, Praxagora déplore que les pauvres n'aient même pas de quoi se faire enterrer ${ }^{52}$. Aussi, entre autres manifestations d'altruisme, était-il de bon ton de fournir l'argent pour les funérailles des concitoyens dans le besoin

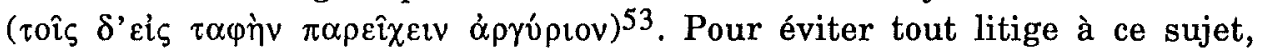
l'Athénien prévoyant veillait à ce que les dépenses fussent prélevées sur son héritage ${ }^{54}$. Cette pratique devint courante à partir de la fin du IVe siècle, et Epicure lui-même avait prévu dans son testament des fonds pour les

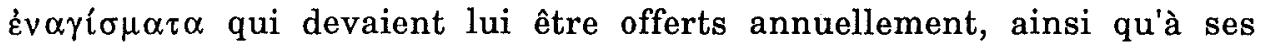
proches ${ }^{55}$. Mais ceci ne concerne plus les funérailles proprement dites.

En quoi consistait dans l'Athènes classique «ce qu'il faut pour être enseveli», comme dit Aristophane? Mises à part les quelques allusions dont il a été question plus haut, nous ne connaissons pas les prix fixés par la loi de Solon, mais pour la fin du Ve et le IVe siècle, Lysias, Platon et Démosthène nous fournissent des renseignements précieux. La mère de Philon, nous dit Lysias, n'avait aucune confiance en son fils et n'osa pas lui confier le soin de ses funérailles : c'est à Antiphanès, qui n'était pas de sa famille, qu'«elle

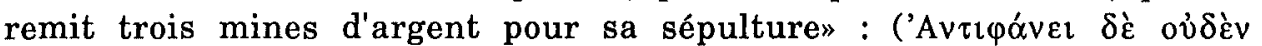

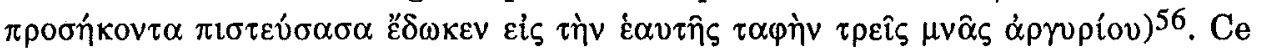

51 R. GARLAND, art. cit., p. 132, pose les jalons d'une «sociology of Attic periboloi». Cf. aussi B. D'Agostino-A. SchnapP, Les morts entre l'objet et l'image, in La mort, les morts..., p. 17 sq.; P. AleXANDREScu, in Dacia, 17 (1973), p. 9 : «les nécropoles représentent un champ propice à une recherche qui nous mènerait vers une sociologie des cimetières».

52 ARIsToph., Ass. fem., 592; cf. aussi Plout., 556 : le pauvre a beau économiser, «il ne laisse même pas ce qu'il faut pour être enseveli» : Acharn., 691.

53 LYS., XIX, 59. Le même précepte se retrouve en Chine, dans le traité rituel sur la conduite idéale de l'homme de bien inséré dans les Entretiens de Confucius, X, 15 : «S'il arrive qu'un de ses amis meure sans avoir de parents pour subvenir aux frais d'enterrement, il dit : «je me charge des obsèques".

54 E.g., IsÉE, VIII, 25 ( On voit dans ce procès que participer aux funérailles et aux frais de l'enterrement prouve la parenté avec le défunt et donc le droit à l'héritage.

55 DIOG. LAËR., X, 10, 18. Pour d'autres exemples semblables à l'époque hellénistique, cf. E. RoHDE, op. cit., p. 205, n. $2 ; 212$, n. $1 ; 540$, n. 3 : des collegia funeratica; A. FESTUGIÈRE, Epicure et ses dieux, 19853 , p. 31-35; W. KAMPS, Les origines de la fondation cultuelle dans la Grèce ancienne, extrait des AHDO, 1 (1937), p. 1-35.

56 LYS., XXXI, 20-21 et 23 : Antiphanès, «ayant reçu l'argent, l'a ensevelie». 
chiffre concorde avec ceux que prescrit Platon dans ses Lois : le philosophe législateur se souvenait de l'enseignement de son maître Socrate qui avait manifesté une indifférence totale quant au traitement réservé à son cadavre, car seul le sort de l'âme lui importait 57 . Contrairement au vaniteux Hippias pour qui le beau consistait entre autres à «recevoir de ses enfants de beaux et

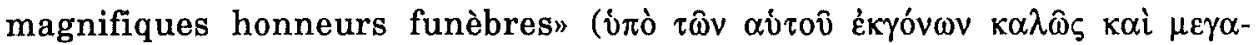
$\lambda \circ \pi \rho \varepsilon \pi \hat{\omega} \zeta \tau \alpha \varphi \hat{\eta} \vee \alpha \iota)^{58}$, Platon considérait qu'il ne faut pas «ruiner sa maison» en s'imaginant que ce "tas de chairs que l'on enterre» est notre être véritable. A l'égard de ce corps sans vie, le législateur prescrira de "ne faire que des frais mesurés" ( $\left.\tau \dot{\alpha} \alpha \mu^{\prime} \tau \rho \imath \alpha \dot{\alpha} v \alpha \lambda i ́ \sigma \kappa o v \tau \alpha\right)^{59}$, et il fixera la valeur maximale des dépenses :

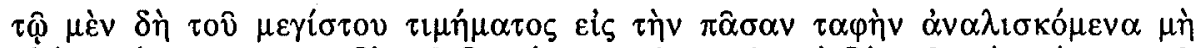

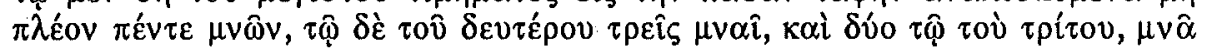

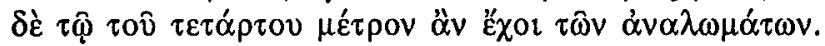

«pas plus de cinq mines ne seront dépensées au total pour la sépulture du citoyen de cens supérieur, trois mines pour le second cens, deux pour le troisième et une pour le quatrième, telle sera la juste mesure des dépenses» 60 .

La juste mesure : voilà le nouveau mot d'ordre que la religion et le droit ont opposé aux anciennes pratiques familiales taxées d'hubris. La dépense de la mère de Philon aurait été jugée tout à fait raisonnable par Platon, mais tout le monde n'était pas aussi économe et certains empruntaient de l'argent pour offrir à leurs parents de dignes funérailles. Un client de Démosthène se

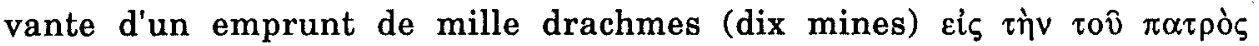
$\tau \alpha \varphi \eta v^{61}$. Il est surprenant de retrouver cette somme chez Platon lui-même. En effet, parmi les lettres que la tradition lui attribue, la treizième présente le

57 Plat., Phéd., 115c-116a. Xénophon prête à Cyrus sur son lit de mort le même désir d'obtenir des obsèques simples, cf. Cyrop., VIII, 7, 25. Pourtant, le Roi avait accordé au vaillant Abradatas des funérailles dignes d'un héros en faisant égorger en son honneur «des boeufs, des chevaux et du petit bétail», cf. VII, 3, 7 .

58 PLAT., Hipp. maj., 291d-e, 292e, 293a.

59 PLAT., Lois, XII, 959c.

60 Plat., Lois, XII, 959d. Noions toutefois que Platon proposait d'accorder aux principaux magistrats des honneurs annuels tout particuliers (cf. XII, 947b sq.) avec des concours musicaux, gymniques et hippiques, qui ne sont pas sans rappeler les honneurs auxquels avaient droit les héros morts pour la patrie, cf. Ménex., 249a sq., et les jeux funèbres de l'épopée homérique. Dans la Rép., III, 413e-414a; V, 465d-e; VII, $540 \mathrm{~b}-\mathrm{c}$, il prévoyait des honneurs semblables pour les bons gardiens, les euthunoi, et les philosophes.

61 DEM., XL, 52. 
philosophe comme un quémandeur qui soutire de l'argent à Denys de Syracuse : il compte sur le tyran pour doter ses petites-nièces et pour payer le tombeau de sa mère, quand elle mourra. On s'accorde en général pour y voir un apocryphe, mais le texte n'en reste pas moins intéressant, car il cite la somme demandée pour le tombeau :

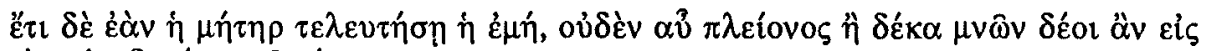

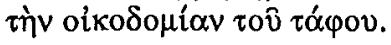

«si ma mère venait à mourir, il ne me faudrait pas plus de dix mines pour la construction du tombeau» (XIII, 361e).

Ce passage peut être interprété dans deux directions opposées. En faveur de l'inauthenticité de la lettre, on peut alléguer qu'il serait pour le moins étonnant que Platon ait voulu consacrer à la sépulture de sa mère le double de la somme qu'il préconisait pour les citoyens de la première classe censitaire. Mais, en faveur de l'authenticité, on peut soutenir que - puisque d'après les événements qu'elle cite, la lettre est censée avoir été écrite en 366 - en l'écrivant, Platon n'avait pas encore réfléchi à ce qu'il prescrira quelques années plus tard dans les Lois $^{62}$.

Un ultime renseignement nous est fourni par Lysias dans son Contre Diogiton (XXXII, 21). Lorsque Diodote mourut en 409, son frère Diogiton fut chargé de l'érection du tombeau et de l'éducation des deux fils du défunt. Poursuivi en justice, Diogiton prétendit avoir dépensé pour le mnèma la somme colossale de cinq mille drachmes, soit cinquante mines. Mais la partie adverse soutenait qu'il n'avait même pas dépensé la moitié de cette

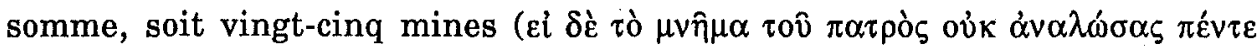

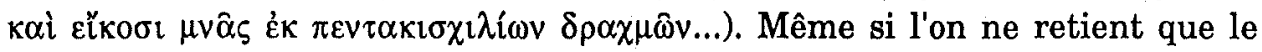
second chiffre, moins de deux mille cinq cents drachmes, ce prix reste exorbitant par rapport aux précédents. Soyons sûrs qu'il est exagéré, tout comme est exagérée la somme que Diogiton prétend avoir déboursée pour l'entretien des deux orphelins : cinq oboles par jour ${ }^{63}$. En effet, la solde d'un marin à l'époque de la guerre du Péloponnèse pouvait varier entre trois oboles et une drachme par journée de travail; un obole par jour devait suffire pour la nourriture d'un adulte ${ }^{64}$. De fait, Diogiton, qui a spolié les deux enfants de leurs $\pi \alpha \tau \rho \omega^{6} \alpha^{65}$, a toutes les raisons de grossir les chiffres dans sa

62 Cf. J. SouILHé, dans sa notice à la lettre XIII de l'édition de la Coll. Univ. France (1960), p. LXXIII.

63 LYS., XXXII, 20.

64 Thuc., VIII, 29; G. GLOTZ, op. cit., p. 337 sq.; A.H.M. JONES, op. cit., p. 135.

65 LYS., op. cit., 10; 22. 
défense ${ }^{66}$. Mais il est vrai aussi que l'affirmation excessive devait rester dans les limites du crédible pour le jury du tribunal.

Quoi qu'il en soit, nous pouvons conclure d'après l'ensemble de ces textes qu'une sépulture relativement modeste devait coûter de une à trois ou même cinq mines, tandis qu'un monument luxueux revenait à dix mines ou plus encore. A quoi il faut ajouter les faux frais du rituel funéraire dont j'ai parlé plus haut. Rappelons simplement ces deux chiffres : vers 400 , la loi des Labyades de Delphes limitait à trente-cinq drachmes les dépenses pour les entaphia, et la loi de Iulis de la fin du Ve siècle fixait à cent drachmes maximum le coût des trois himatia du mort.

A titre de comparaison, nous savons qu'un manteau pouvait coûter jusqu'à vingt drachmes au début du IVe siècle ${ }^{67}$. Or, les prix avaient sans doute augmenté entre le Ve et le IVe siècle ${ }^{68}$ : les cent drachmes autorisées par la loi de Iulis restent donc élevées. On peut également se faire une idée du coût du sacrifice funéraire que prescrivait la loi de Solon et qu'autorisait celle de Iulis. Un bœuf revenait approximativement à cinq drachmes du temps de Solon ${ }^{69}$, mais il coûtait déjà en moyenne quarante à cinquante drachmes vers 400 , et son prix augmenta encore durant le IVe siècle jusqu'à septante et même nonante drachmes. Quant au mouton, autre victime sacrificielle des rites funéraires, il se payait de dix à dix-sept drachmes au IVe siècle ${ }^{70}$.

On réalise aisément que l'addition de tous ces frais ait pu être qualifiée par certains de gaspillage. C'est contre de telles extravagances que réagirent

66 Aussi R. Garland a-t-il peut-être tort de s'en tenir à cette seule donnée pour montrer que l'érection d'un peribolos «was clearly a very costly undertaking», art. cit., p. 130, et J.K. DAVIES, Athenian propertied families, 1971, p. 154 (cité par R. Garland) : «This is an astonishing amount for erecting a funerary monument even if we accept the lower figure of 2500 drachmas (...) and casts considerable light on the values and priorities of Athenian society".

67 A.H.M. JoNES, Athenian democracy, 1957, p. 135; ARISTOPH., Plout., 982-983.

68 G. GLOTZ, Le travail dans la Grèce antique, 1920, p. 233-234 (prix des esclaves aux Ve et IVe siècles); p. 285 (hausse des prix : de Solon à Alexandre, les prix ont sextuplé).

69 G. GLOTZ, op. cit., p. 285.

70 Ces prix mentionnés dans les calendriers sacrés d'Attique ont été réunis et comparés il y a peu par F. VAN STRATEN, Greek sacrificial representations : livestock prices and religious mentality, in Gifts to the Gods. Proceedings of the Uppsala Symposium 1985, Uppsala, 1987 (Boreas Acta Universitatis Upsaliensis, Uppsala Studies in Ancient Mediterranean and Near Eastern Civilizations, 15), p. 159-170, en part. p. 166 , où l'auteur dresse un tableau des prix des bovins, des ovins, des porcins et des caprins. 
les législateurs et qu'Euripide écrivit : «Les hommes sont fous de faire tant de vaines dépenses pour les morts» 71 .

A côté de la loi panhellénique, «loi divine, loi non-écrite» 72 , qui exigeait que l'on rende les derniers devoirs aux défunts, il y avait donc les lois écrites qui imposaient la modestie des funérailles et interdisaient les gaspillages. On sait que le Grec craignait par-dessus tout de rester ataphios ou anosios mais, en plus, il voulait que son taphos - terme qui désigne aussi bien la tombe que les funérailles - soit luxueux. Après la mort, le monument funéraire devient un véritable substitut du corps : il perpétue sa présence parmi les vivants. C'est pourquoi personne ne se contentait des quelques mottes de terre symboliques dont Antigone recouvrit pieusement le corps de son frère.

Rue de l'Église, 18

Xavier DE SCHUTTER

B - 1630 LINKKBEEK

71 EUR., fr. 641 Nauck.

72 EUR., Suppl., 524-527; 670; SopH., Aj., 1129-1131; Ant., 453 sq.; 1113. 